\title{
Identification of aberrantly methylated differentially expressed genes targeted by differentially expressed miRNA in osteosarcoma
}

\author{
Ting-Xuan Wang ${ }^{1 \#}$, Wen-Le Tan ${ }^{2 \#}$, Jin-Cheng Huang ${ }^{3 \#}$, Zhi-Fei Cui ${ }^{1}$, Ri-Dong Liang ${ }^{4}$, Qing-Chu Li ${ }^{5}$, \\ Hai Lu ${ }^{1}$
}

${ }^{1}$ Department of Orthopedics, The Fifth Affiliated Hospital of Sun Yat-sen University, Sun Yat-sen University, Zhuhai 519000, China; ${ }^{2}$ Department of Orthopedics, Luoding People's Hospital, Luoding 527200, China; ${ }^{3}$ Department of Orthopedics, Henan Provincial People's Hospital, People's Hospital of Zhengzhou University, People's Hospital of Henan University, Zhengzhou 450003, China; ${ }^{4}$ Department of Orthopedics, Southern Medical University Affiliated Nanhai Hospital, Southern Medical University, Foshan 523800, China; ${ }^{5}$ Department of Orthopedics, The Third Affiliated Hospital of Southern Medical University, Academy of Orthopedics, Southern Medical University, Guangzhou 510000, China

Contributions: (I) Conception and design: H Lu; (II) Administrative support: H Lu; (III) Provision of study materials or patients: H Lu, QC Li; (IV) Collection and assembly of data: TX Wang, WL Tan, JC Huang; (V) Data analysis and interpretation: TX Wang; (VII) Manuscript writing: All authors; (VII) Final approval of manuscript: All authors.

"These authors contributed equally to this work.

Correspondence to: Hai Lu, PhD. Department of Orthopedics, The Fifth Affiliated Hospital of Sun Yat-sen University, No. 52 East Meihua Road, Xiangzhou District, Zhuhai 519000, China. Email: luhai01@163.com; Qing-chu Li, PhD. Department of Orthopedics, The Third Affiliated Hospital of Southern Medical University, No.183 West Zhongshan Avenue, Tianhe District, Guangzhou 510000, China. Email: lqc16@263.net.

\begin{abstract}
Background: Osteosarcoma (OS) is the most common primary bone tumors diagnosed in children and adolescents. Recent studies have shown a prognostic role of DNA methylation in various cancers, including OS. The aim of this study was to identify the aberrantly methylated genes that are prognostically relevant in OS.

Methods: The differentially expressed mRNAs, miRNAs and methylated genes (DEGs, DEMs and DMGs respectively) were screened from various GEO databases, and the potential target genes of the DEMs were predicted by the RNA22 program. The protein-protein interaction (PPI) networks were constructed using the STRING database and visualized by Cytoscape software. The functional enrichment and survival analyses of the screened genes was performed using the $\mathrm{R}$ software.

Results: Forty-seven downregulated hypermethylated genes and three upregulated hypomethylated genes were identified that were enriched in cell activation, migration and proliferation functions, and were involved in cancer-related pathways like JAK-STAT and PI3K-AKT. Eight downregulated hypermethylated tumor suppressor genes (TSGs) were identified among the screened genes based on the TSGene database. These hub genes are likely involved in OS genesis, progression and metastasis, and are potential prognostic biomarkers and therapeutic targets.

Conclusions: TSGs including PYCARD, STAT5A, CXCL12 and CXCL14 were aberrantly methylated in OS, and are potential prognostic biomarkers and therapeutic targets. Our findings provide new insights into the role of methylation in OS progression.
\end{abstract}

Keywords: Osteosarcoma (OS); methylation; miRNA; tumor suppressor gene (TSG); bioinformatics

Submitted Nov 27, 2019. Accepted for publication Jan 18, 2020.

doi: $10.21037 /$ atm.2020.02.74

View this article at: http://dx.doi.org/10.21037/atm.2020.02.74

\section{Introduction}

Osteosarcoma (OS) is the most common primary malignant bone tumors in children and young adults. It originates from mesenchymal cells and is characterized by the appearance of spindle cells and aberrant osteoid formation. OS can occur in any bone, although the distal femur, proximal tibia and the proximal humerus are the most 
common sites, accounting for respectively $43 \%, 23 \%$ and $10 \%$ of the cases. Within the bone, OS typically affects the metaphysis close to the growth plate (1). OS also has a high metastasis rate of $20 \%$, and commonly invades the lungs and other bones (2).

Studies in recent years have shown that epigenetic changes play an important role in tumorigenesis and progression. DNA methylation involves addition of methyl groups (-CH3) to cytosines in the $\mathrm{CpG}$ dinucleotides, and both hypermethylation or hypomethylation can result in long-term silencing of imprinted genes, transposons and the inactive $\mathrm{X}$ chromosome (3). Aberrant methylation is frequently observed in tumors, and silencing of the promoter regions of tumor suppressor genes (TSGs) by hypermethylation of $\mathrm{CpG}$ islands (4) affects the cell cycle checkpoint, apoptosis, signal transduction, cell adhesion and angiogenesis genes (5). MicroRNAs (miRNAs) are short non-coding RNAs that regulate gene expression at the post-translational level by binding to the 3'-UTR of target mRNA, and are also aberrantly expressed in various cancers (6). Abnormal DNA methylation patterns have been detected in OS that interfere with the TSGs, and promote tumor initiation and progression (7). Although the DNA methylation patterns in OS have gained attention as vital biomarkers and therapeutic targets, the regulatory network between DNA methylation, miRNAs and target genes remains elusive.

Previous studies have identified several differentially methylated genes (DMGs) and differentially expressed genes (DEGs) in OS through bioinformatics analysis (8). However, the regulatory networks of OS-related miRNAs, mRNAs and methylation patterns in OS are largely unknown. To this end, we screened the mRNA, miRNA and gene methylation profiles from OS and normal bone microarray datasets, in order to identify the DEGs, DMGs, TSGs and differentially expressed miRNAs (DEMs) related to OS. The aim of our study was to identify and validate the aberrantly methylated TSGs in OS. Our findings provide new insights into the potential tumorigenic role of abnormal DNA methylation, and identify potential biomarkers and therapeutic targets for OS.

\section{Methods}

\section{Identification of DEGs, DEMs and DMGs}

The miRNA datasets GSE28423 and GSE65071, the mRNA dataset GSE36001 and the methylation dataset
GSE36002 were downloaded from the GEO database (https://www.ncbi.nlm.nih.gov/geo/). The GSE28423 was registered on the GPL8227 microarray platform (Agilent-019118 Human miRNA Microarray 2.0 G4470B) and contained data from 19 OS and 4 normal bone samples. GSE65071 was registered on the microarray platform of GPL19631 (Exiqon human V3 microRNA PCR panel I+II) and included data of 20 OS and 15 normal bone samples. GSE36001 and GSE36002 were from the GPL6102 microarray platform (Illumina human-6 v2.0 expression beadchip), and included data of 19 OS cell lines and 6 normal osteoblasts and bones samples.

GEO2R was used to screen for the DEGs, DEMs and DMGs from the respective datasets using $\mid \log \mathrm{FCl}>1$ or $\log |\beta|>0.2$ (for DMGs) and $\mathrm{P}$ value $<0.05$ as the thresholds. The putative target genes of the overlapping DEMs of GSE28423 and GSE65071 datasets were predicted using the RNA22 tool (https://cm.jefferson.edu/rna22/Interactive/). The expression matrix of the DEGs was analyzed by the GSEA tool according to $\mathrm{P}$ values $<0.05$ and $\mathrm{q}$ values $<0.05$, and the putative TSGs were identified from the TSGene database (https://bioinfo.uth.edu/TSGene/download. cgi). Finally, the Venn diagram tool (http://bioinformatics. psb.ugent.be/webtools/Venn/) was used to determine (I) overlapping hypomethylated mRNAs, up-regulated genes and targets to identify the up-regulated hypomethylated mRNAs; (II) overlapping hypermethylated mRNAs, down-regulated mRNAs and targets for downregulated hypermethylated mRNAs; (III) overlapping down-regulated mRNAs, hypermethylated mRNAs, targets and TSGs to identify the down-regulated hypermethylated TSGs.

\section{Functional analyses of the relevant genes}

Biological process (BP) enrichment analysis and Kyoto Encyclopedia of Genes and Genomes (KEGG) analysis were performed using the ClusterProfiler package of $\mathrm{R}$ software, with $\mathrm{P}<0.05$ as the threshold. Gene set enrichment analysis (GSEA) was conducted using the GSEA desktop tool. The Search Tool for the Retrieval of Interacting Genes (STRING, https://string-db.org/) database was used to construct the PPI networks, which were then visualized using CYTOSCAPE.

\section{Validation of TSGs}

The prognostic value of the 8 TSGs was determined by survival analysis using the online database R2: Genomics 
Analysis and Visualization Platform (http://r2.amc.nl). The clinical data and the expression data were extracted from a dataset, the Mixed Osteosarcoma-Kuijjer-127-vstilmnhwg6v2. The patients were stratified into the respective low- and high-expression groups using the $\mathrm{R} 2$ scan method. The correlation between TSGs methylation and expression was determined by Spearman's rank correlation analysis, with $\mid$ Cor $\mid>0.3$ and $\mathrm{P}<0.05$ as the thresholds.

\section{Cell culture and treatment}

The OS cell line MG63 was purchased from the American Type Culture Collection (ATCC, Manassas, VA, USA), and cultured in DMEM (Gibco, USA) supplemented with 10\% FBS under $5 \% \mathrm{CO}_{2}$ at $37{ }^{\circ} \mathrm{C}$. The DNA methyltransferase inhibitor 5-azacytidine (5-Aza) was purchased from MedChemExpress. The MG63 cells were treated with $5 \mu \mathrm{M} 5$-Aza or DMSO (vehicle) for $48 \mathrm{~h}$.

\section{RNA isolation and quantitative RT-PCR}

Total RNA was extracted from the cells using Total RNA Extraction Reagent (Vazyme, China), and reverse transcribed into cDNA using the HiScript II Q RT SuperMix (Vazyme, China). RT-PCR was conducted using ChamQ SYBR qPCR Master Mix (Vazyme, China) in a Lightcycler 96 (Roche, Switzerland). The relative mRNA expression levels were determined by the $2^{-\Delta \Delta C T}$ method. All tests were performed three times, and GAPDH was used as the internal control. The primer sequences are shown in Table S1.

\section{Cell proliferation assay}

MG63 cells were seeded into a 96 well plate at the density of $3 \times 10^{3} /$ well, and cultured with DMSO or 5-Aza for $24,48,72$, 96 and $120 \mathrm{~h}$. The medium was replaced with fresh medium supplemented with $10 \mu \mathrm{L}$ Cell Counting Kit-8 (CCK-8) reagent/well (DOJINDO, Japan), and the cells were incubated for another hour. The absorbance at $450 \mathrm{~nm}$ was measured using a microplate reader (Bio.Tek, USA), and the proliferation rates were calculated after subtracting the background absorbance. The experiment was performed thrice.

\section{Wound-healing assay}

MG63 cells were seeded into 6 well plates at $80 \%$ density and treated with $5 \mu \mathrm{M} 5$-Aza or DMSO for $48 \mathrm{~h}$. The monolayer was scratched perpendicular to the plate with a sterile $200 \mu \mathrm{l}$ pipette tip. The dislodged cells were removed by washing twice with PBS, and serum-free medium was added. The cells were cultured for $24 \mathrm{~h}$, and the wound edges were photographed at the same position at $0,24 \mathrm{~h}$ under an inverted microscope. The migration rate was determined based on the width of the wound edges. The assay was performed in triplicate.

\section{Invasion assay}

In vitro invasion was analyzed using 24 well Invasion Chambers (8 $\mu \mathrm{m}$ pore size; Costar, Corning, USA). For the invasion assay, the transwell chambers were pre-coated with $50 \mu \mathrm{L}$ Matrigel (Corning, USA) for $3 \mathrm{~h}$. MG63 cells were seeded into the upper chambers at the density of $1 \times 10^{4} / 100 \mu \mathrm{L}$ in serum free medium, and the lower chambers were filled with $500 \mu \mathrm{L}$ DMEM supplemented with $10 \%$ FBS. After incubating for $24 \mathrm{~h}$, the cells were washed twice with PBS and fixed with $4 \%$ paraformaldehyde for 30 minutes at room temperature. The PET membranes were air-dried and stained with $0.1 \%$ crystal violet for 15 minutes. The non-invaded cells were wiped off, and the invaded cells were photographed and counted under an inverted microscope in five random fields per membrane. Both assays were performed in triplicate.

\section{Statistical analyses}

All statistical analyses were performed using GRAPHPAD PRISM7 (GraphPad Prism Software Inc., San Diego, CA, USA) or SPSS 23.0. Two groups were compared using Student's $t$-test with Welch correction in case of significantly different variance, or multiple groups were compared using one-way ANOVA analysis. $\mathrm{P}<0.05$ was considered statistically significant.

\section{Results}

\section{Identification of DEGs and DEMs in microarray}

Workflow of how aberrantly methylated differentially expressed genes were identified was shown in Figure 1. A total of 15 DEMs were identified in the OS samples from the GSE28423 and GSE65071 microarray datasets (see methods), and the heat maps are shown in Figure $2 A, B$. In addition, 835 DEGs (Table S2) were also identified from the GSE36001 dataset, of which 574 were downregulated and 261 were up-regulated (Figure 2C). Finally, 4,313 DMGs were detected in the GSE36002 dataset (Figure 2D), of which 3,441 were hypermethylated and 872 


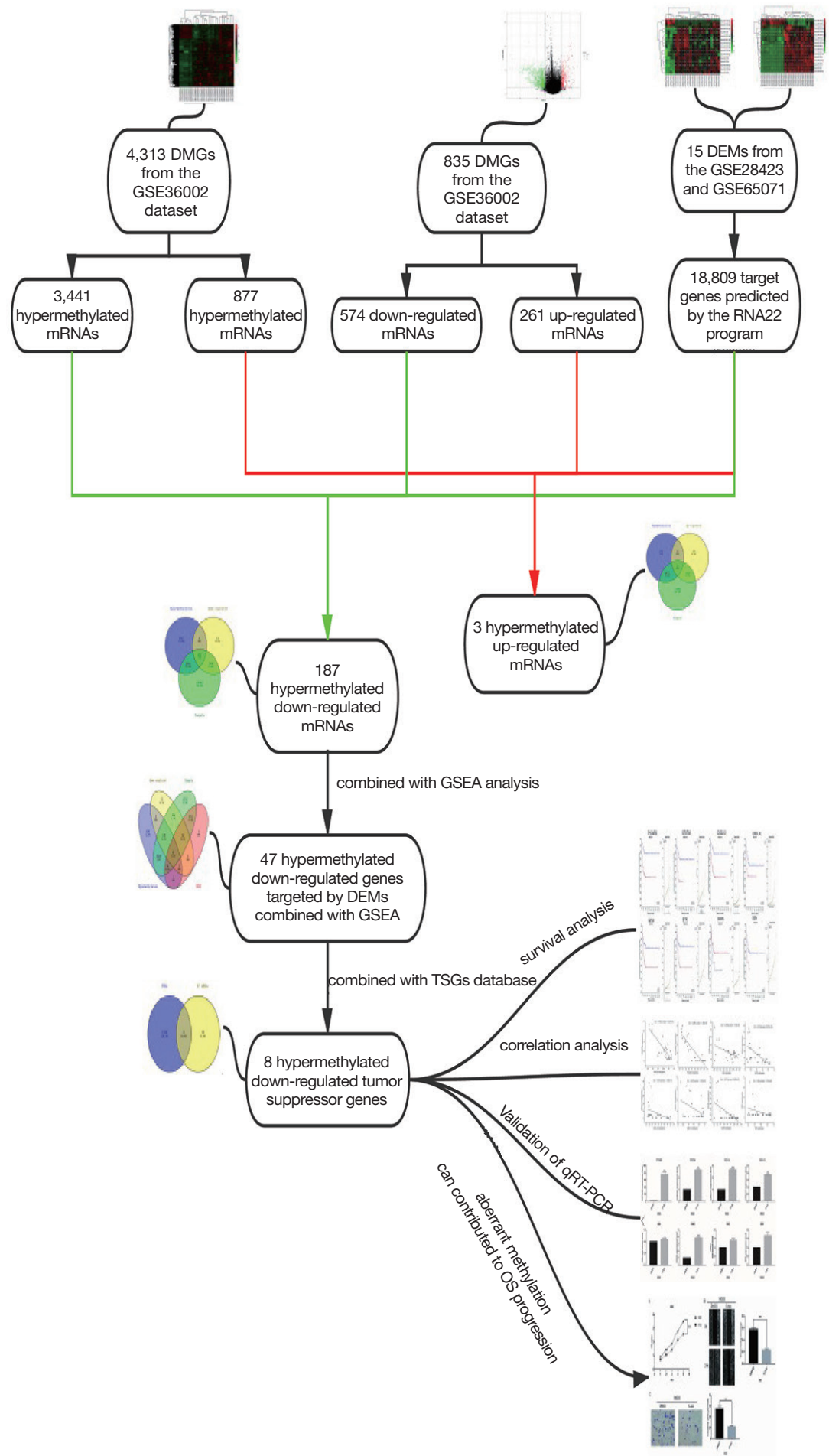

Figure 1 Workflow of how aberrantly methylated differentially expressed genes were identified. 

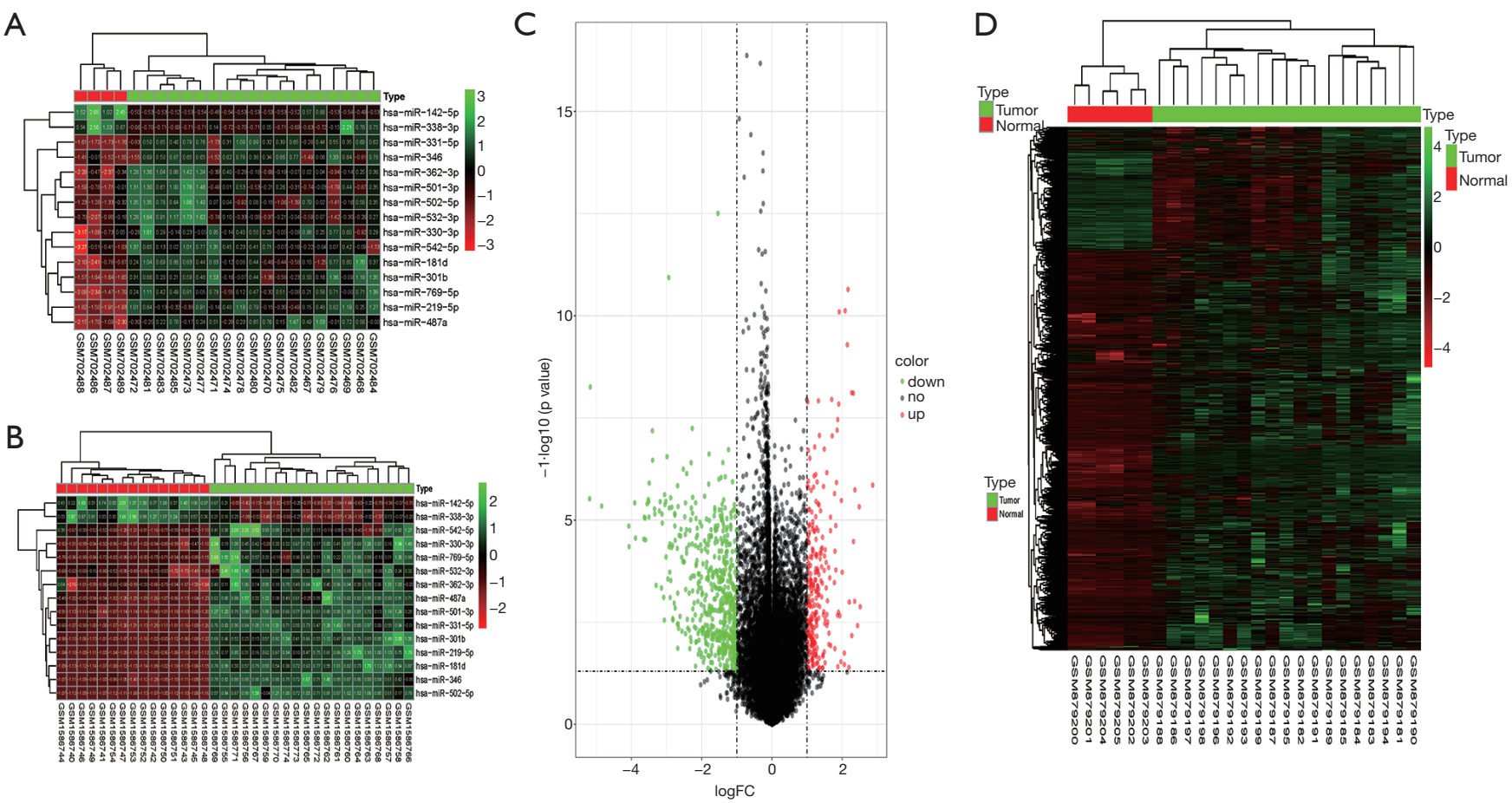

Figure 2 Identification of DEGs, DEMs and DMGs in OS. (A) Heatmap of 15 DEMs in GSE28423; (B) heatmap of 15 DEMs in GSE65071; (C) volcano plot showing DEG distribution in GSE36001; (D) heatmap of DMGs in GSE36002. The black area, green area and the red area represent the non-DEGs, down-regulated mRNAs, and up-regulated mRNAs, respectively. DEGs, differentially expressed genes; DEMs, differentially expressed miRNAs; DMGs, differentially methylated genes.

were hypomethylated.

\section{Identification of aberrantly methylated DEGs targeted by the DEMs}

The RNA22 program predicted 18,809 target genes of the 15 DEMs. The overlap between these targets, DEGs and DMGs revealed 187 hypermethylated down-regulated genes (Figure $3 A$ ) and only 3 hypomethylated up-regulated genes (Figure 3B). The latter included BMP4, PYCR1 and PRAME, which are involved in BP promoting cancers and other diseases. We surmised therefore that BMP4, PYCR1 and PRAME were significant in OS progression. The DEMs and 187 hypermethylated down-regulated genes are respectively shown in Tables $S 3, S 4$.

\section{Identification of bypermethylated down-regulated genes targeted by DEMs combined with GSEA}

To further identify the DEGs in OS, we conducted GSEA based on the GSE36001 dataset. Ten KEGG gene sets was significantly enriched in OS compared to normal tissues, including a total of 539 mRNAs according to $\mathrm{P}$ values $<0.05$ and $q$ values $<0.05$ (Figure $4 A$ ). The most significantly enriched KEGG gene set in OS was the KEGG_SPLICEOSOME (Figure 4B), and its constituent DEGs are visualized in Figure $4 C$. Other significant gene sets included KEGG_CELL CYCLE and KEGG_DNA REPLICATION (Figure S1). Overlapping of the 539 mRNAs of KEGG gene sets, DEGs, DMGs and 18,809 target genes of the 15 DEMs, revealed 47 hypermethylated down-regulated genes (Figure 4D) that are likely involved in OS. The list of 47 hypermethylated down-regulated genes was shown in Table S5.

\section{Functional enrichment analysis and PPI network construction}

Gene ontology (GO) analysis and KEGG analysis were next performed to determine the $\mathrm{BP}$ and pathways associated with the 47 hypermethylated downregulated genes in OS (Figure 5). As shown in Figure 5C, the most enriched $\mathrm{BP}$ were positive regulation of response to external stimulus (GO:0032103) and phagocytosis (GO:0006909). 
A

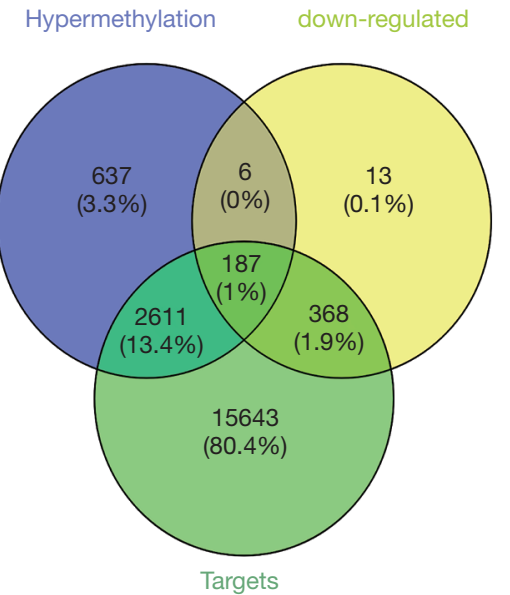

B Hypomethylation up-regulated

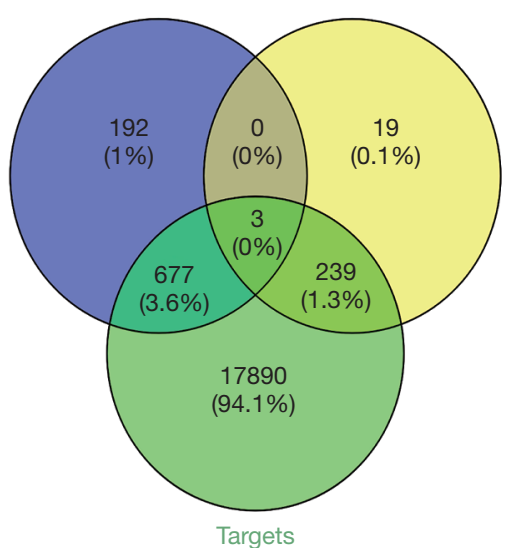

Figure 3 Identification of aberrantly methylated DEGs. (A) A total of 193 hypermethylated down-regulated genes were identified, of which 187 genes were targets of DEMs; (B) three hypomethylated up-regulated genes targeted by DEMs were identified. DEGs, differentially expressed genes; DEMs, differentially expressed miRNAs.
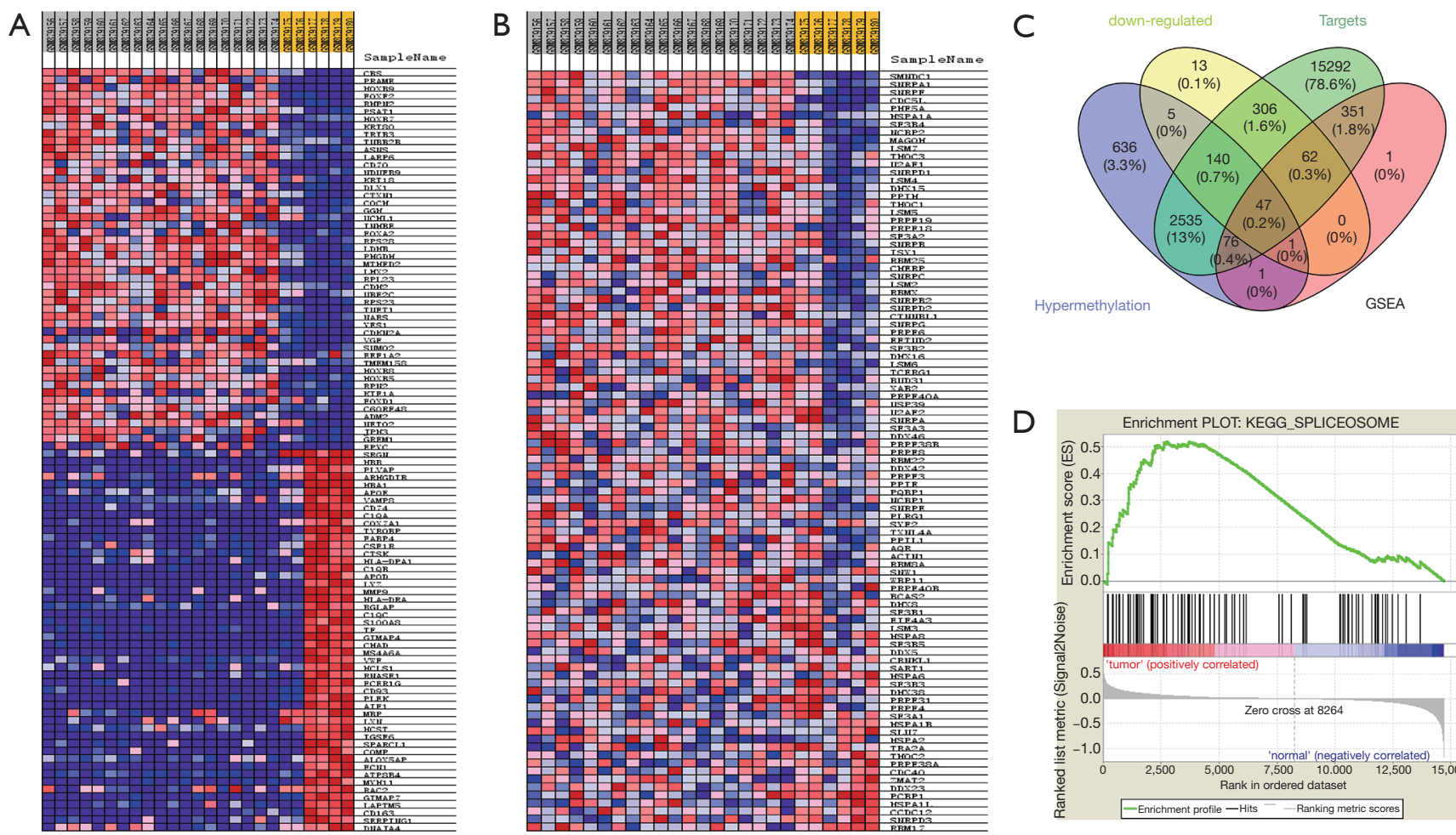

Figure 4 GSEA results. (A) Heatmap of DEGs from GSE36001; (B) heatmap of the "KEGG_SPLICEOSOME" gene set; (C) Venn diagram showing the overlap between hypermethylated down-regulated genes and GSEA results; (D) enrichment plot of KEGG_SPLICEOSOME. The top portion of plots show the enrichment scores for each gene, and the bottom portion shows the ranked genes. Y-axis: ranking metric, $\mathrm{X}$-axis: individual ranks for all genes. GSEA, gene set enrichment analysis; DEGs, differentially expressed genes. 
A

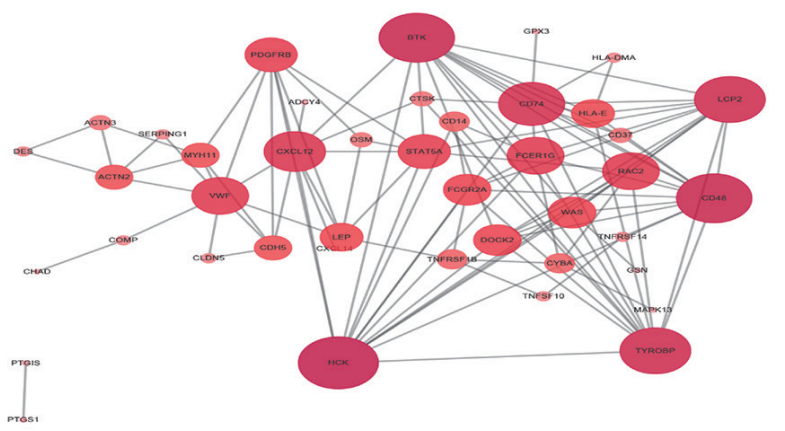

B

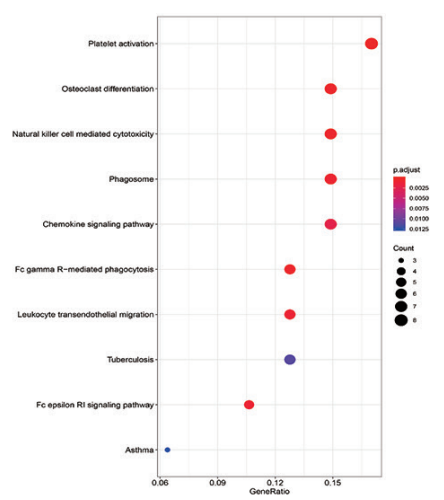

C
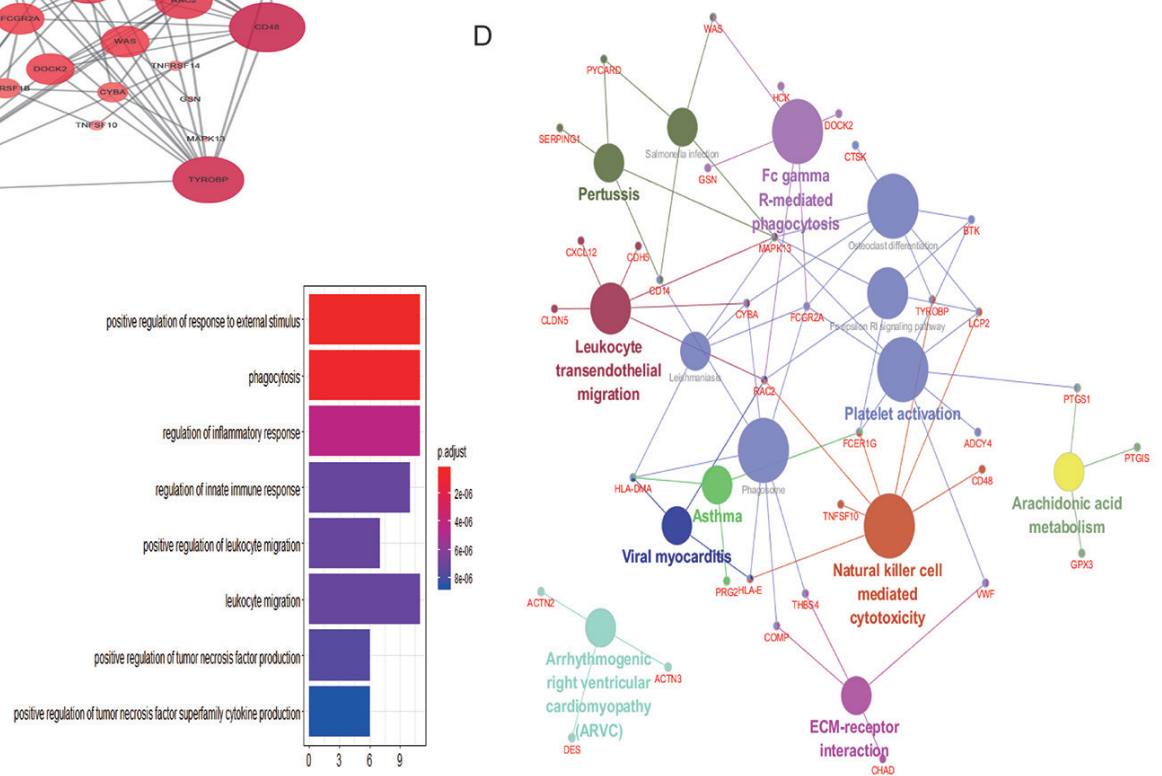

Figure 5 Functional analysis and PPI network of the hypermethylated down-regulated genes. (A) PPI network of 47 hypermethylated downregulated genes visualized by the Cytoscape software. The size of the dots and the gradation of color indicate the strength of interaction; (B) bubble chart shows the significant pathways. The color depth indicates statistical significance, Y-axis represents the KEGG pathway, X-axis represents the proportion of enriched genes, and the size of the points indicates the number of genes; (C) bar graph showing the significantly enriched biological processes in the DEGs. The color depth indicates statistical significance, the Y-axis shows the GO-BP terms and $\mathrm{X}$-axis represents the proportion of enriched genes; (D) clue GO analysis results. The large points and small points represent the significant KEGG pathways the enriched genes, respectively. PPI, protein-protein interaction; KEGG, Kyoto Encyclopedia of Genes and Genomes; DEGs, differentially expressed genes; GO, gene ontology; BP, biological process.

Furthermore, KEGG pathway enrichment analysis showed that most genes were enriched in platelet activation (hsa04611), osteoclast differentiation (hsa04380), natural killer cell mediated cytotoxicity (hsa04650), phagosome (hsa04145), and chemokine signaling pathway (hsa04062). The top 10 significant pathways for the screened genes are shown in Figure 5B, indicating that the DEGs are likely involved in some osteoclast differentiation pathways and several cancer-related pathways, such as the JAK-STAT and PI3K-Akt. Furthermore, the clue GO analysis showed that MAPK13 and FCGR2A were significantly enriched in more pathways compared to the other DEGs (Figure 5D). The PPI network further showed 40 nodes with strong correlations and 2 nodes with weak correlations (Figure 5A), indicating complex interactions between the downregulated and hypermethylated mRNAs at the protein level.

\section{Function analysis and PPI network construction of aberrantly methylated TSGs involved in OS}

Our previous study showed that down-regulated hypermethylated mRNAs play an important role in tumorigenesis. Therefore, we screened for known TSGs among the 47 OS-related DEGs, and identified eight (Figure 6A). This indicated that aberrant methylation of these TSGs downregulated their expression levels in OS and promoted tumorigenesis. PPI network construction showed that 7 down-hypermethylated TSGs were strongly correlated at the protein level (Figure 6B). Further GO analysis showed that the key genes were mainly associated with cell regulation, migration and apoptosis 
A

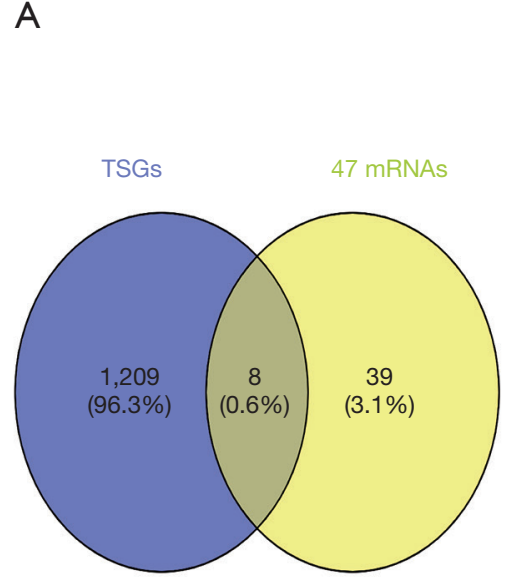

B

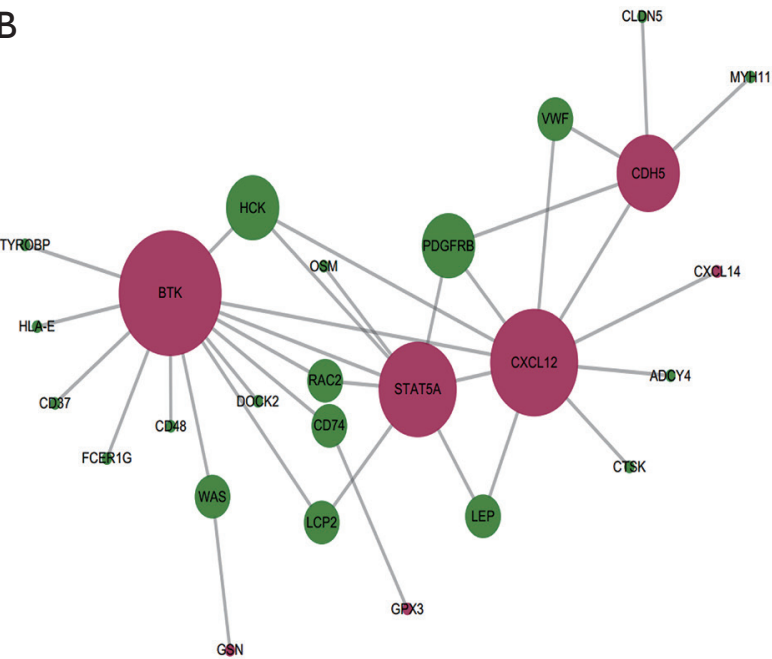

Figure 6 Identification of TSGs. (A) Venn diagram showing the aberrantly methylated DEGs in the TSGs database. The overlapping area includes 8 hypermethylated down-regulated TSGs; (B) hub genes highlighted in the PPI network of hypermethylated down-regulated genes. The 7 key genes are highlighted and shown as the hub genes in the network. Red region and green region indicate TSGs, and nonTSGs, respectively. DEGs, differentially expressed genes; TSGs, tumor suppressor genes; PPI, protein-protein interaction.

(Table 1), and pathway enrichment analysis indicated that they were significantly enriched in the NF-kappa B signaling pathway, chemokine signaling pathway and viral carcinogenesis (Table 2). Therefore, these aberrantly methylated TSGs are likely involved in OS progression, and are potential biomarkers.

\section{Prognostic assessment of the down-regulated bypermethylated TSGs in OS}

To determine the prognostic value of the above 8 TSGs in OS, we assessed the metastasis free survival of patients from the dataset, Mixed OS (Mesenchymal)-Kuijjer-127vst-ilmnhwg6v2, after stratifying them into the respective low- and high-expressing groups. Patients expressing low levels of BTK, GPX3, CXCL12, CXCX14, PYCARD and STAT5A had worse metastasis free survival compared to the corresponding high expression groups (Figure 7), indicating that these genes have a significant impact on patient prognosis.

\section{Correlation between methylation and gene expression}

Correlation analysis of the gene expression and DNA methylation data revealed a significant inverse correlation between DNA methylation and the respective gene expression levels (Figure 8). The methylation sites and correlation coefficient of the significant TSGs are shown in Table 3. The most significant effect of DNA methylation was seen on the expression levels of STAT5A and PYCARD (Cor $=-0.826, \mathrm{P}$ value $=5.207 \mathrm{e}-20 ;$ Cor $=-0.887, \mathrm{P}$ value $=1.468 \mathrm{e}-18)$.

\section{Inbibition of DNA methylation can upregulate the key genes}

To further validate the impact of methylation on the expression of the TSGs, we treated MG63 cells with the methyltransferase inhibitor 5-Aza, and examined the expression levels of the relevant genes. As shown in Figure 9, PYCARD, STAT5A, CDH5, CXCL12 and CXCL14 mRNA levels were significantly increased after 5-Aza treatment, thereby confirming the in silico data. In contrast, BTK and GPX3 expression was not significantly affected by inhibiting DNA methylation.

\section{Aberrant methylation contributed to OS progression}

The findings so far indicated that methylated TSGs were involved in cancer-related pathways and contributed to OS progression. To further elucidate the effect of aberrant methylation on OS progression, the MG63 cells were treated with 5-Aza and various functional assays were performed. Blocking DNA methylation not only decreased the survival of the OS cells in vitro (Figure 10A), but also 
Table 1 Significant biological processes in which the 8 hub genes were mainly involved

\begin{tabular}{|c|c|c|c|c|}
\hline ID & Description & $P$ value & Gene & Count \\
\hline GO:2000401 & Regulation of lymphocyte migration & $1.71 \mathrm{E}-06$ & PYCARD/CXCL12/CXCL14 & 3 \\
\hline GO:0072676 & Lymphocyte migration & $9.76 \mathrm{E}-06$ & PYCARD/CXCL12/CXCL14 & 3 \\
\hline GO:0002687 & Positive regulation of leukocyte migration & 1.49E-05 & PYCARD/CXCL12/CXCL14 & 3 \\
\hline GO:0032878 & Regulation of establishment or maintenance of cell polarity & $4.50 \mathrm{E}-05$ & CDH5/GSN & 2 \\
\hline GO:0008064 & Regulation of actin polymerization or depolymerization & 4.74E-05 & PYCARD/CXCL12/GSN & 3 \\
\hline GO:0030832 & Regulation of actin filament length & 4.82E-05 & PYCARD/CXCL12/GSN & 3 \\
\hline GO:0002685 & Regulation of leukocyte migration & 4.90E-05 & PYCARD/CXCL12/CXCL14 & 3 \\
\hline GO:0098760 & Response to interleukin-7 & $7.57 \mathrm{E}-05$ & STAT5A/BTK & 2 \\
\hline GO:0098761 & Cellular response to interleukin-7 & 7.57E-05 & STAT5A/BTK & 2 \\
\hline GO:2000404 & Regulation of $\mathrm{T}$ cell migration & 0.00013 & PYCARD/CXCL12 & 2 \\
\hline GO:0110053 & Regulation of actin filament organization & 0.00015 & PYCARD/CXCL12/GSN & 3 \\
\hline GO:0032103 & Positive regulation of response to external stimulus & 0.00022 & CXCL12/CXCL14/BTK & 3 \\
\hline GO:0072678 & T cell migration & 0.00028 & PYCARD/CXCL12 & 2 \\
\hline GO:0032956 & Regulation of actin cytoskeleton organization & 0.0003 & PYCARD/CXCL12/GSN & 3 \\
\hline GO:0002532 & $\begin{array}{l}\text { Production of molecular mediator involved in inflammatory } \\
\text { response }\end{array}$ & 0.00032 & PYCARD/BTK & 2 \\
\hline GO:0007015 & Actin filament organization & 0.00051 & PYCARD/CXCL12/GSN & 3 \\
\hline GO:0002690 & Positive regulation of leukocyte chemotaxis & 0.00056 & CXCL12/CXCL14 & 2 \\
\hline GO:2000106 & Regulation of leukocyte apoptotic process & 0.0006 & CXCL12/BTK & 2 \\
\hline GO:0050727 & Regulation of inflammatory response & 0.00062 & PYCARD/CDH5/BTK & 3 \\
\hline GO:0030838 & Positive regulation of actin filament polymerization & 0.00076 & PYCARD/GSN & 2 \\
\hline GO:0008630 & $\begin{array}{l}\text { Intrinsic apoptotic signaling pathway in response to DNA } \\
\text { damage }\end{array}$ & 0.00083 & PYCARD/CXCL12 & 2 \\
\hline GO:0051249 & Regulation of lymphocyte activation & 0.00084 & PYCARD/GSN/BTK & 3 \\
\hline GO:0050900 & Leukocyte migration & 0.00087 & PYCARD/CXCL12/CXCL14 & 3 \\
\hline GO:0002821 & Positive regulation of adaptive immune response & 0.00087 & PYCARD/BTK & 2 \\
\hline GO:0071887 & Leukocyte apoptotic process & 0.00091 & CXCL12/BTK & 2 \\
\hline GO:0002688 & Regulation of leukocyte chemotaxis & 0.00094 & CXCL12/CXCL14 & 2 \\
\hline
\end{tabular}

Table 1 (continued) 
Table 1 (continued)

\begin{tabular}{llll}
\hline ID & Description & P value & Gene \\
\hline G0:0015696 & Ammonium transport & 0.00094 & CXCL12/BTK \\
G0:0090066 & Regulation of anatomical structure size & 0.00098 & PYCARD/CXCL12/GSN \\
GO:0001938 & Positive regulation of endothelial cell proliferation & 0.00099 & STAT5A/CXCL12 \\
\hline
\end{tabular}

GO, Gene Ontology; PYCARD, PYD and CARD domain containing; STAT5A, signal transducer and activator of transcription 5A; CDH5, Cadherin 5; CXCL12, C-X-C Motif Chemokine Ligand 12; CXCL14, C-X-C Motif Chemokine Ligand 14; GSN, Gelsolin; BTK, Bruton tyrosine kinase; GPX3, glutathione peroxidase 3.

Table 2 Significant KEGG pathways in which the 8 hub genes were mainly involved.

\begin{tabular}{llcc}
\hline ID & Description & P value & Gene \\
\hline hsa04064 & NF-kappa B signaling pathway & 0.004273 & CXCL12/BTK \\
hsa04670 & Leukocyte transendothelial migration & 0.005333 & CDH5/ CXCL12 \\
hsa04217 & Necroptosis & 0.010906 & STAT5A/PYCARD \\
hsa04062 & Chemokine signaling pathway & 0.0148017 & CXCL12/CXCL14 \\
hsa05203 & Viral carcinogenesis & 0.0164769 & STAT5A/GSN \\
hsa04810 & Regulation of actin cytoskeleton & 0.0185588 & CXCL12/GSN \\
hsa04060 & Cytokine-cytokine receptor interaction & 0.033662 & 2 \\
hsa05340 & Primary immunodeficiency & 0.0370792 & CXCL12/CXCL14 \\
hsa04672 & Intestinal immune network for IgA production & 0.0488436 & BTK \\
\hline
\end{tabular}

KEGG, Kyoto Encyclopedia of Genes and Genomes; PYCARD, PYD And CARD Domain Containing; STAT5A, Signal Transducer And Activator Of Transcription 5A; CDH5, Cadherin 5; CXCL12, C-X-C Motif Chemokine Ligand 12; CXCL14, C-X-C Motif Chemokine Ligand 14; GSN, Gelsolin; BTK, Bruton Tyrosine Kinase; GPX3, Glutathione Peroxidase 3.

markedly diminished their migration (Figure 10B) and invasion capacities (Figure 10C) compared to the DMSO controls. Taken together, aberrant DNA methylation is conducive to OS progression, and inhibiting DNA methyltransferases can decrease proliferation, migration and invasion of OS cells.

\section{Discussion}

OS is a primary bone malignancy with a high rate of recurrence. Despite advancements in surgery and chemotherapy, the overall survival rates have been dismal over the past 20 years (9). Aberrant DNA methylation is an epigenetic modification that is frequently observed in most cancers, and is a major risk factor that drives tumorigenesis via gene silencing (7). Since epigenetic modifications affect gene functions without altering the DNA sequence, they result in more diverse gene expression profiles (10). Novel molecular biology techniques and bioinformatics integration analysis in recent years have enabled identification of aberrant methylation patterns in various cancers, and provided insights into the underlying mechanisms.

We identified 187 hypermethylated genes and 3 hypomethylated genes in OS based on mRNA, miRNA and methylation datasets, along with 15 DEMs that are relevant in OS and other cancers except has-miR-331-5p (11). The hsa-miR-142-5p (12), hsa-miR-338-3p (13) and hsa-miR$542-5 \mathrm{p}$ were in particular strongly associated with OS, and can help identify the key genes involved in OS progression.

BMP4, PYCR1 and PRAME were the 3 hypomethylated upregulated genes in OS. BMP4 is a secreted ligand of the TGF- $\beta$ superfamily that recruits and activates SMAD family transcription factors, and is involved in cardiac development and adipogenesis (14). BMP4 promoter hypomethylation is associated with worse prognosis in gastric cancer (15), indicating that the aberrant methylation of BMP4 in OS is 


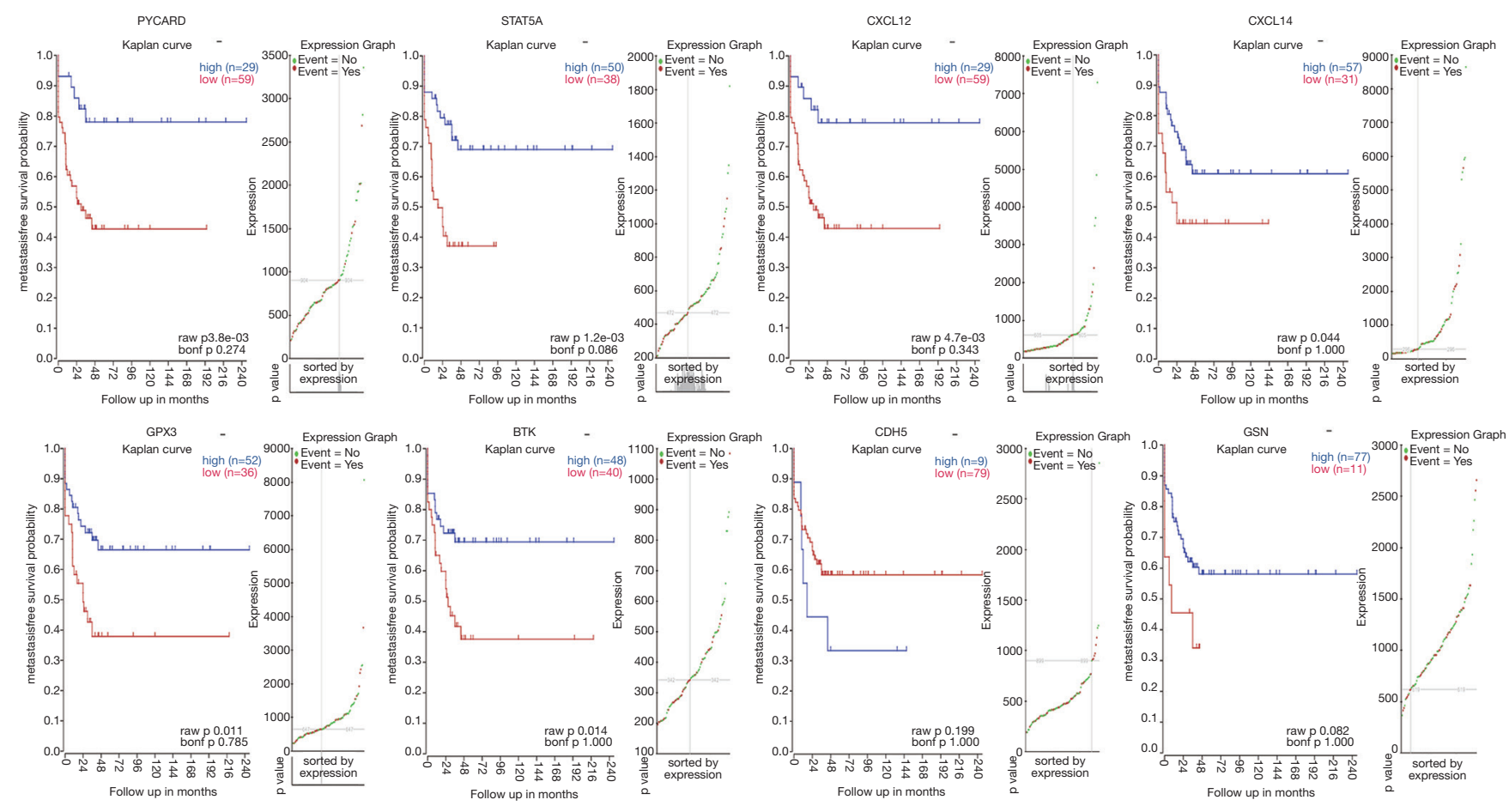

Figure 7 Prognostic relevance of hypermethylated TSGs in OS. Kaplan-Meier survival analysis of 8 hub genes were conducted by R2: Genomics Analysis and Visualization Platform (http://r2.amc.nl). The differences were tested using the log-rank test. P values are demonstrated in the lower right corner of each image and the numbers of samples with high expression and low expression are displayed in the higher right corner of each image. TSGs, tumor suppressor genes; Bonf p: Adjusted P value for multiple comparisons (Bonferroni method); Raw P: raw P value.

likely oncogenic. PYCR1 catalyzes NAD(P)H-dependent conversion of pyrroline-5-carboxylate to proline during cell proliferation and metabolism, and is up-regulated in a wide range of cancers. Overexpression of PYCR1 contributes to poor prognosis in prostate cancer (16), and based on our results, may have a pro-tumorigenic role in OS. PRAME is overexpressed in melanoma, myeloid leukemia, neuroblastoma (17), and head and neck cancer, and its hypomethylated form promotes the progression of chronic myeloid leukemia (18). In OS as well, PRAME overexpression is associated with poor prognosis, and increases tumor cell proliferation by attenuating cell cycle arrest (19). Taken together, these genes likely promote OS progression, although the underlying mechanisms need to be elucidated. Furthermore, they are potential prognostic biomarkers and therapeutic targets in OS.

Forty-seven hypermethylated down-regulated genes in OS were enriched in functions like response to external stimulus, cell migration and cell proliferation, chemotaxis and inflammatory response, all of which contribute to tumor metastasis (20). Aberrant migration and proliferation is an important cellular program in tumors, and are mediated by dysregulated signaling pathways (21). KEGG analysis revealed that the OS-related hypermethylated genes were significantly associated with the PI3K-Akt and JAT-STAT pathways. The former is the most frequently mutated network in human cancers, and is also dysregulated in tumors due to methylation (22). It promotes cancer cell survival by inhibiting pro-apoptotic and activating anti-apoptotic genes, Furthermore, the downstream mTOR kinase also promotes cell growth and protein synthesis, and downregulates this pathway through a feedback loop. Constitutive activation of the PI3K-Akt pathway is often accompanied by loss or mutations in the tumor suppressor PTEN (23). It also plays a critical role in OS genesis by inhibiting apoptosis and activating pro-survival pathways (24). The JAK/STAT pathway is also dysregulated in many solid tumors and increases tumor cell proliferation and angiogenesis, resulting in worse prognosis (25). Tyrosine phosphorylation and nuclear localization of the STATs have been observed in the 

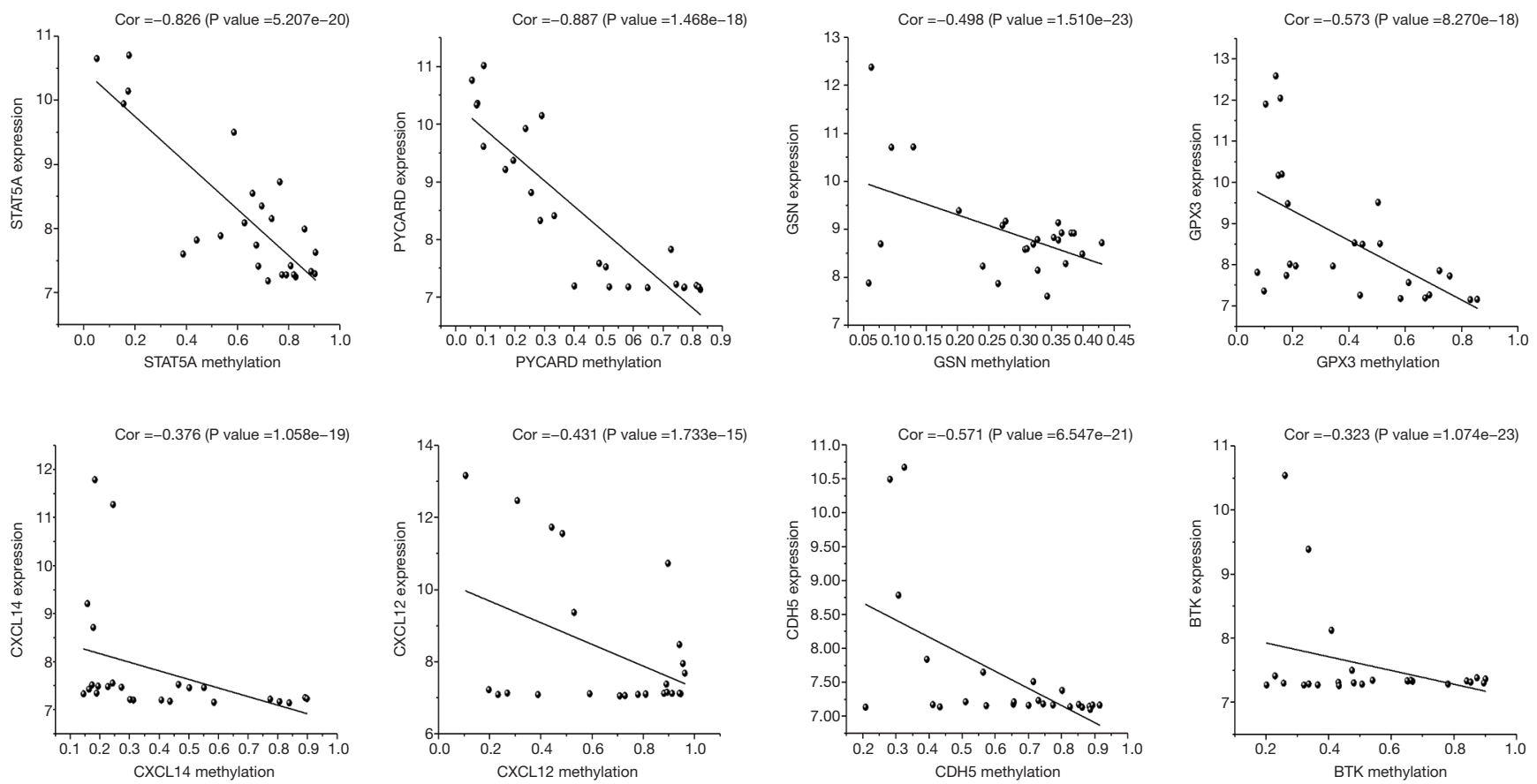

Figure 8 The correlation between methylation values and expression values of 8 hub genes. Higher correlation coefficient indicates stronger association between gene expression and methylation. Cor, correlation coefficient.

Table 3 The methylation sites and correlation coefficient of The TSGs.

\begin{tabular}{|c|c|c|c|}
\hline Gene symbol & Methylation site & Correlation & $P$ value \\
\hline STAT5A & cg03001305 & -0.826 & $5.207 e-20$ \\
\hline $\mathrm{CDH} 5$ & cg22319147 & -0.571 & $6.547 e-21$ \\
\hline CXCL12 & cg18618334 & -0.431 & $1.733 e-15$ \\
\hline GSN & cg17071957 & -0.498 & $1.510 \mathrm{e}-23$ \\
\hline GPX3 & cg17820459 & -0.573 & $8.270 e-18$ \\
\hline BTK & cg03791917 & -0.323 & $1.074 \mathrm{e}-23$ \\
\hline
\end{tabular}

TSGs, tumor suppressor genes; PYCARD, PYD And CARD Domain Containing; STAT5A, Signal Transducer And Activator Of Transcription 5A; CDH5, Cadherin 5; CXCL12, C-X-C Motif Chemokine Ligand 12; CXCL14, C-X-C Motif Chemokine Ligand 14; GSN, Gelsolin; BTK, Bruton Tyrosine Kinase; GPX3, Glutathione Peroxidase 3.

tumor tissues across a range of cancers, indicating that JAK/ SAT activation correlates with worse prognosis. We next established a clue GO network of the hypermethylated genes and these pathways, and observed significant enrichment of MAPK13 and FCGR2A. The latter is an IgG receptor, and methylation of its promoter decreases binding affinity to the human IgG2, which is associated with higher susceptibility to Kawasaki Disease (26). The p38 MAP kinase MAPK13 is aberrantly expressed in several tumors, and its promoter methylation contributes to melanoma progression (27). However, the role of MAPK13 and FCGR2A in OS remains to be elucidated.

The hypermethylated downregulated genes included 8 TSGs-CDH5, BTK, GPX3, PYCARD, CXCL12, CXCL14, 
PYCARD

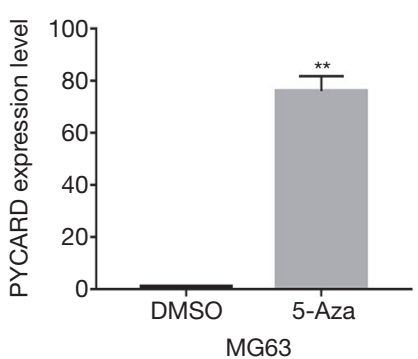

GSN

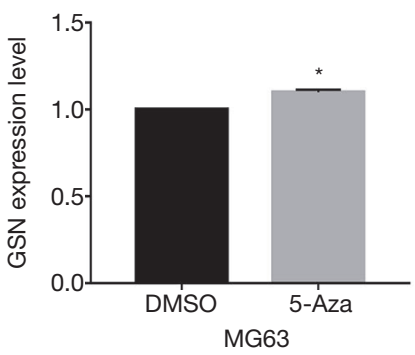

STAT5A

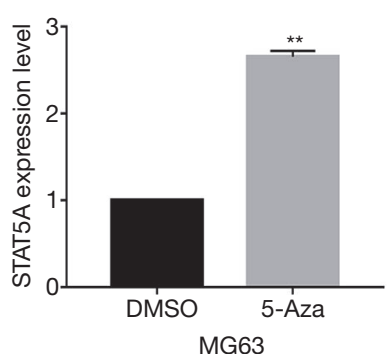

$\mathrm{CDH} 5$

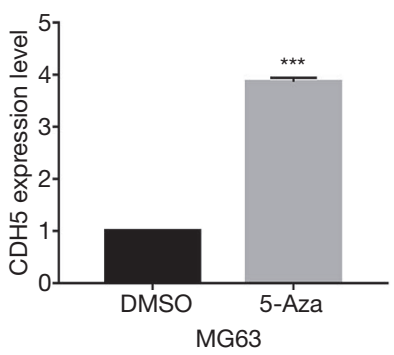

CXCL14

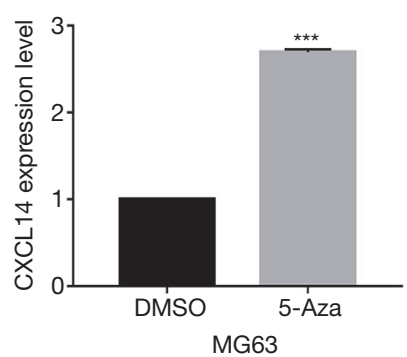

GPX3

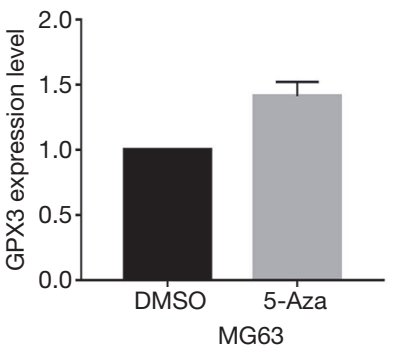

CXCL12

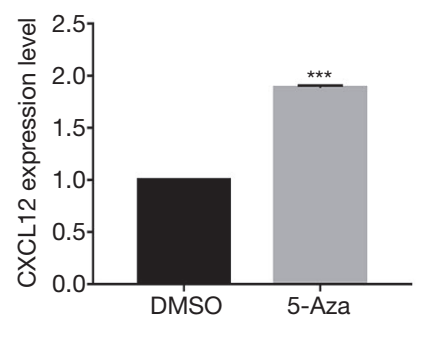

BTK

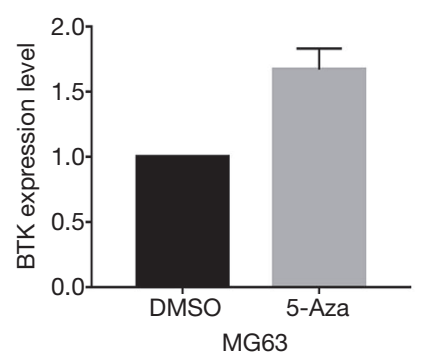

Figure 9 Expression levels of the key genes with 5-Aza treatment in MG63 cells. Error bars represent SEM, ${ }^{* * *}, \mathrm{P}<0.001 ;{ }^{* *}, \mathrm{P}<0.01$; *, $\mathrm{P}<0.05$. 5-Aza, 5-azacytidine.

A

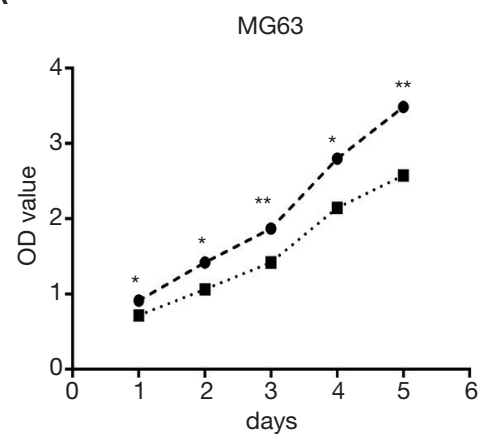

C

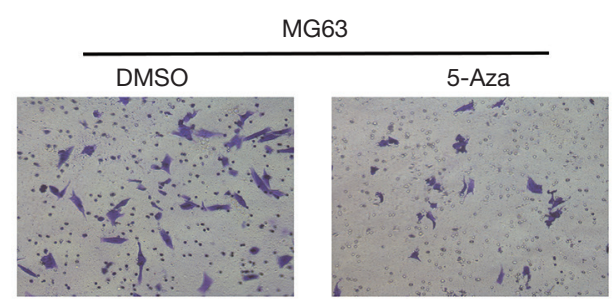

B
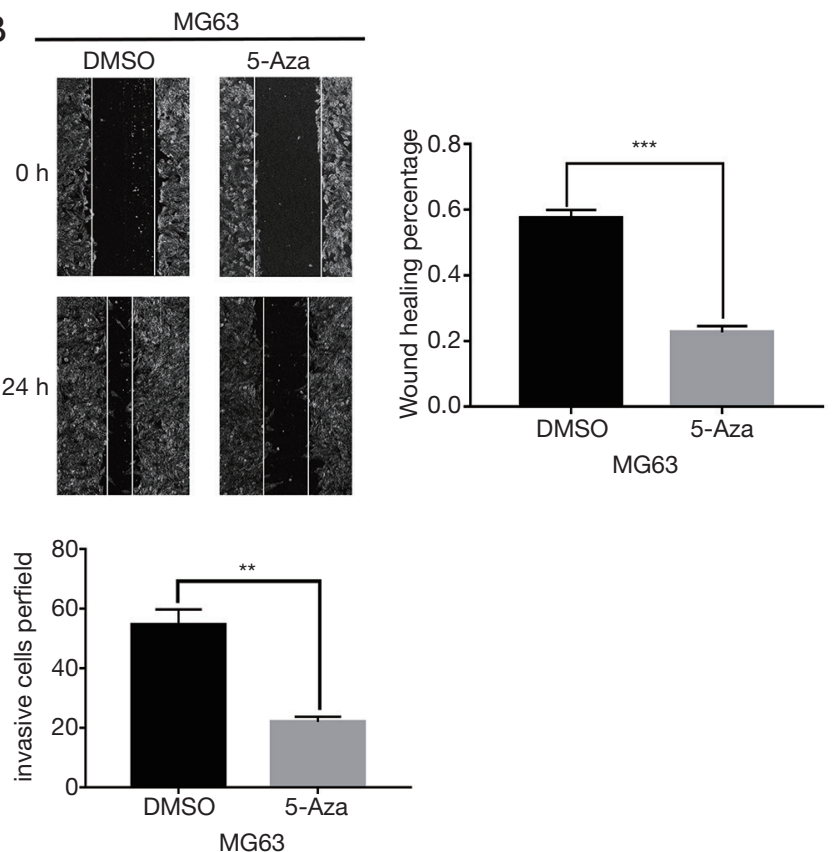

Figure 10 Aberrant methylation contributed to OS progression. (A) After 5-Aza treatment, the proliferation of MG63 cells were significantly decreased; the Wound-healing assay (B) and Transwell Migration Assay (C) evaluated that the migration and invasion of MG63 cells were significantly decreased after 5-Aza treatment compared to those of the control group. $0.1 \%$ crystal violet, scale bar, 100 or 200 $\mu \mathrm{m}$, error bars represent SEM; ${ }^{* * *}, \mathrm{P}<0.001$; ${ }^{* *}, \mathrm{P}<0.01 ;{ }^{*}, \mathrm{P}<0.05$. 5-Aza, 5-azacytidine. 
STATSA and GSN. PYCARD is a signaling factor consisting of a PYD and CARD domain, and mediates the apoptotic pathway by activating caspases (28). Studies show that hypermethylation-mediated silencing of PYCARD enables tumor cell survival by blocking apoptosis (29). We showed a strong correlation between PYCARD hypermethylation and down-regulation in OS, and verified that its low expression levels indicated worse prognosis based on clinical data. Furthermore, 5-Aza treatment markedly upregulated PYCARD, which strongly suggests that the aberrant methylation of PYCARD drives OS genesis. CXCL12 is a chemokine of the intercrine family, and binds with CXCR4 to initiate divergent pathways related to chemotaxis and cell survival (30). High expression levels of CXCL12 is associated with poor prognosis in ovarian cancer (31), and increased migration and invasiveness of adamantinomatous craniopharyngiomas (32). In contrast, down-regulation of CXCL12 have also been detected in OS via promoter hypermethylation by DNA methyltransferase 1 (DNMT1) (33). According to our findings, low expression and hypermethylation of CXCL12 indicates worse prognosis in OS, which make it a potential therapeutic target. CXCL14 is another chemokine involved in immunoregulatory and inflammatory processes (34), as well as tumor migration and invasion. It inhibited colorectal cancer cell migration by suppressing NF-kB signaling, whereas hypermethylationmediated silencing promoted migration (35). BTK is a component of the Toll-like receptors (TLR) pathway, and promotes inflammatory responses (36). In addition, it is a modulator of $\mathrm{p} 53$ that can be induced in response to DNA damage and p53 activation, and phosphorylates the latter to enhance apoptosis (37). We found that low expression levels of BTK due to hypermethylation was associated with poor prognosis in OS.

CDH5 belongs to the cadherin superfamily, and is involved in the vasculogenic mimicry of glioblastoma stemlike cells under hypoxic conditions (38). Low expression levels of $\mathrm{CDH} 5$ mediated by promoter methylation was strongly associated with poor overall survival in neuroblastomas (39). In agreement with this, $\mathrm{CDH} 5$ was expressed at low levels in OS tissues compared to normal samples, and predicted poor prognosis. STAT5A is a transcription factor that is frequently dysregulated in cancer. Methylation-dependent promoter region silencing of STAT5A inhibited NPM1ALK expression in ALK + TCL cell lines (40). One study reported low levels of STAT5A in OS and correlated it with increased tumor progression and worse overall survival (41). Consistent with this, the hypermethylation status of STAT5A was strongly associated with its low expression levels as well as poor prognosis in our study. Interestingly, BTK, CXCL12, CDH5 and STAT5A showed strong functional connectivity at the protein level, although the molecular mechanisms remain to be elucidated.

GPX3 is a glutathione peroxidase that protects cells against ROS and DNA damage, and is an established tumor suppressor in various cancers (42). Gene silencing of GPX3 by promoter hypermethylation has been reported in hepatocellular carcinoma (43), and similar trends were observed in the OS samples as well. GSN is an actinbinding protein that regulates actin filament formation and disassembly, as well as apoptosis via DNase I binding and release (44). GSN downregulation via promoter methylation predicts poor survival in gastric cancer (45), whereas overexpression of GSN promoted growth and invasion of OS cells (46). This contradicts our findings that hypermethylation and down regulation of GSN likely drive OS progression.

To summarize, we identified several TSGs that were hypermethylated and downregulated in the OS tissues, indicating a strong relationship between DNA methylation and tumorigenesis. Although our findings have to be validated in experimental studies and the underlying mechanisms also need to be elucidated, we can conclude that the aforementioned genes are significantly involved in OS progression, and are potential prognostic markers and/ or therapeutic targets. There are however several limitations in this study. Since the CpG sites information was not available, the significance of specific methylation sites of these hub genes could not be determined. Furthermore, we did not correlate the expression data with the clinical parameters in the same datasets. Further studies are needed to validate the role of these TSGs in OS.

\section{Conclusions}

We identified 47 hypermethylated down-regulated mRNAs targeted by significant miRNAs in OS, of which 8 are established tumor suppressors. The aberrantly methylated TSGs may be the potential prognostic biomarkers and therapeutic targets for OS. Our findings provide new insights into the role of methylation in OS progression.

\section{Acknowledgments}

The authors thank the GEO databases for the availability of the data. 
Funding: This study was supported by grants from the National Natural Science Foundation of China (Grant number 81572628, 81372869), The Science and Technology Project of Guangzhou (Grant No. 201704020033) and The Science and Technology Project of Tianhe District, Guangzhou (Grant No. 201604KW012).

\section{Footnote}

Conflicts of Interest: The authors have no conflicts of interest to declare.

Ethical Statement: The authors are accountable for all aspects of the work in ensuring that questions related to the accuracy or integrity of any part of the work are appropriately investigated and resolved.

Open Access Statement: This is an Open Access article distributed in accordance with the Creative Commons Attribution-NonCommercial-NoDerivs 4.0 International License (CC BY-NC-ND 4.0), which permits the noncommercial replication and distribution of the article with the strict proviso that no changes or edits are made and the original work is properly cited (including links to both the formal publication through the relevant DOI and the license). See: https://creativecommons.org/licenses/by-nc-nd/4.0/.

\section{References}

1. Gill J, Ahluwalia MK, Geller D, et al. New targets and approaches in osteosarcoma. Pharmacol Ther 2013;137:89-99.

2. Yan GN, Lv YF, Guo QN. Advances in osteosarcoma stem cell research and opportunities for novel therapeutic targets. Cancer Lett 2016;370:268-74.

3. Wu SC, Zhang Y. Active DNA demethylation: many roads lead to Rome. Nat Rev Mol Cell Biol 2010;11:607-20.

4. Cui J, Wang W, Li Z, et al. Epigenetic changes in osteosarcoma. Bull Cancer 2011;98:E62-8.

5. Suzuki H, Maruyama R, Yamamoto E, et al. DNA methylation and microRNA dysregulation in cancer. MOL ONCOL 2012;6:567-78.

6. Zhuang M, Qiu X, Cheng D, et al. MicroRNA-524 promotes cell proliferation by down-regulating PTEN expression in osteosarcoma. Cancer Cell Int 2018;18:114.

7. Li X, Lu H, Fan G, et al. A novel interplay between HOTAIR and DNA methylation in osteosarcoma cells indicates a new therapeutic strategy. J Cancer Res Clin
Oncol 2017;143:2189-200.

8. Kresse SH, Rydbeck H, Skårn M, et al. Integrative analysis reveals relationships of genetic and epigenetic alterations in osteosarcoma. PLoS One 2012;7:e48262.

9. O'Day K, Gorlick R. Novel therapeutic agents for osteosarcoma. Expert Rev Anticancer Ther 2009;9:511-23.

10. Moore LD, Le T, Fan G. DNA Methylation and Its Basic Function. Neuropsychopharmacology 2013;38:23-38.

11. Xie B, Ding Q, Han H, et al. miRCancer: a microRNAcancer association database constructed by text mining onliterature. Bioinformatics 2013;29:638-44.

12. Jones KB, Salah Z, Del Mare S, et al. miRNA Signatures Associate with Pathogenesis and Progression of Osteosarcoma. Cancer Res 2012;72:1865-77.

13. Cao R, Shao J, Hu Y, et al. microRNA-338-3p inhibits proliferation, migration, invasion, and EMT inosteosarcoma cells by targeting activator of $90 \mathrm{kDa}$ heat shock protein ATPasehomolog 1. Cancer Cell Int 2018;18:49.

14. Gustafson B, Hammarstedt A, Hedjazifar S, et al. BMP4 and BMP Antagonists Regulate Human White and Beige Adipogenesis. Diabetes 2015;64:1670-81.

15. Ivanova $\mathrm{T}$, Zouridis $\mathrm{H}, \mathrm{Wu} \mathrm{Y}$, et al. Integrated epigenomics identifies BMP4 as a modulator of cisplatin sensitivity in gastric cancer. Gut 2013;62:22-33.

16. Zeng T, Zhu L, Liao M, et al. Knockdown of PYCR1 inhibits cell proliferation and colony formation via cell cycle arrest and apoptosis in prostate cancer. Med Oncol 2017;34:27.

17. Oberthuer A, Hero B, Spitz R, et al. The tumor-associated antigen PRAME is universally expressed in high-stage neuroblastoma and associated with poor outcome. Clin Cancer Res 2004;10:4307-13.

18. Roman-Gomez J, Jimenez-Velasco A, Agirre X, et al. Epigenetic regulation of PRAME gene in chronic myeloid leukemia. Leuk Res 2007;31:1521-8.

19. Tan P, Zou C, Yong B, et al. Expression and prognostic relevance of PRAME in primary osteosarcoma. Biochem Biophys Res Commun 2012;419:801-8.

20. Lu H, Ouyang W, Huang C. Inflammation, a key event in cancer development. Mol Cancer Res 2006;4:221-33.

21. Zhou N, Si Z, Li T, et al. Long non-coding RNA CCAT2 functions as an oncogene in hepatocellular carcinoma, regulating cellular proliferation, migration and apoptosis. Oncol Lett 2016;12:132-8.

22. Chang L, Graham PH, Ni J, et al. Targeting PI3K/ Akt/mTOR signaling pathway in the treatment of prostate cancer radioresistance. Crit Rev Oncol Hematol 
2015;96:507-17.

23. Stemke-Hale K, Gonzalez-Angulo AM, Lluch A, et al. Integrative genomic and proteomic analysis of PIK3CA, PTEN, and AKT mutations in breast cancer. Cancer Res 2008;68:6084-91.

24. Zhang J, Yu XH, Yan YG, et al. PI3K/Akt signaling in osteosarcoma. Clin Chim Acta 2015;444:182-92.

25. Lai SY, Johnson FM. Defining the role of the JAK-STAT pathway in head and neck and thoracic malignancies: Implications for future therapeutic approaches. Drug Resist Updat 2010;13:67-78.

26. Kuo HC, Hsu YW, Wu MS, et al. FCGR2A Promoter Methylation and Risks for Intravenous Immunoglobulin Treatment Responses in Kawasaki Disease. Mediators Inflamm 2015;2015:564625.

27. Gao L, Smit MA, van den Oord JJ, et al. Genomewide promoter methylation analysis identifies epigenetic silencing of MAPK13 in primary cutaneous melanoma. Pigment Cell Melanoma Res 2013;26:542-54.

28. McConnell BB, Vertino PM. Activation of a caspase-9mediated apoptotic pathway by subcellular redistribution of the novel caspase recruitment domain protein TMS1. Cancer Res 2000;60:6243-7.

29. Conway KE, McConnell BB, Bowring CE, et al. TMS1, a novel proapoptotic caspase recruitment domain protein, is a target of methylation-induced gene silencing in human breast cancers. Cancer Res 2000;60:6236-42.

30. Teicher BA, Fricker SP. CXCL12 (SDF-1)/CXCR4 Pathway in Cancer. CLIN Cancer Res 2010;16:2927-31.

31. Guo Q, Gao BL, Zhang XJ, et al. CXCL12-CXCR4 Axis Promotes Proliferation, Migration, Invasion, and Metastasis of Ovarian Cancer. Oncol Res 2014;22:247-58.

32. Yin X, Liu Z, Zhu P, et al. CXCL12/CXCR4 promotes proliferation, migration, and invasion of adamantinomatous craniopharyngiomas via PI3K/AKT signal pathway. J Cell Biochem 2019;120:9724-36.

33. Li B, Wang Z, Wu H, et al. Epigenetic Regulation of CXCL12 Plays a Critical Role in Mediating Tumor Progression and the Immune Response In Osteosarcoma. Cancer Res 2018;78:3938-53.

34. Schwarze SR, Luo J, Isaacs WB, et al. Modulation of CXCL14 (BRAK) expression in prostate cancer. Prostate 2005;64:67-74.

35. Cao B, Yang Y, Pan Y, et al. Epigenetic Silencing of CXCL14 Induced Colorectal Cancer Migration and Invasion. Discov Med 2013;16:137-47.
36. Liu X, Zhan Z, Li D, et al. Intracellular MHC class II molecules promote TLR-triggered innate immune responses by maintaining activation of the kinase Btk. Nat Immunol 2011;12:416-24.

37. Althubiti M, Rada M, Samuel J, et al. BTK Modulates p53 Activity to Enhance Apoptotic and Senescent Responses. Cancer Res 2016;76:5405-14.

38. Mao XG, Xue XY, Wang L, et al. CDH5 is specifically activated in glioblastoma stemlike cells and contributes to vasculogenic mimicry induced by hypoxia. Neuro Oncol 2013;15:865-79.

39. Fujita T, Igarashi J, Okawa ER, et al. CHD5, a tumor suppressor gene deleted from 1p36.31 in neuroblastomas. J Natl Cancer Inst 2008;100:940-9.

40. Zhang Q, Wang H, Liu X, et al. STAT5A is epigenetically silenced by the tyrosine kinase NPM1-ALK and acts as a tumor suppressor by reciprocally inhibiting NPM1-ALK expression. NAT MED 2007;13:1341-8.

41. Guo Z, Tang Y, Fu Y, et al. Decreased expression of STAT5A predicts poor prognosis in osteosarcoma. PATHOL RES PRACT 2019;215:519-24.

42. Barrett CW, Ning W, Chen $X$, et al. Tumor suppressor function of the plasma glutathione peroxidase gpx 3 in colitis-associated carcinoma. Cancer Res 2013;73:1245-55.

43. Cao S, Yan B, Lu Y, et al. Methylation of promoter and expression silencing of GPX3 gene in hepatocellular carcinoma tissue. Clin Res Hepatol Gastroenterol 2015;39:198-204.

44. Yeh YL, Hu WS, Ting WJ, et al. Hypoxia Augments Increased HIF-1 $\alpha$ and Reduced Survival Protein p-Akt in Gelsolin (GSN)-Dependent Cardiomyoblast Cell Apoptosis. Cell Biochem Biophys 2016;74:221-8.

45. Wang HC, Chen CW, Yang CL, et al. Tumor-Associated Macrophages Promote Epigenetic Silencing of Gelsolin through DNA Methyltransferase 1 in Gastric Cancer Cells. Cancer Immunol Res 2017;5:885-97.

46. Ma X, Sun W, Shen J, et al. Gelsolin promotes cell growth and invasion through the upregulation of $\mathrm{p}-\mathrm{AKT}$ and p-P38 pathway in osteosarcoma. Tumour Biol 2016;37:7165-74.

Cite this article as: Wang TX, Tan WL, Huang JC, Cui ZF, Liang RD, Li QC, Lu H. Identification of aberrantly methylated differentially expressed genes targeted by differentially expressed miRNA in osteosarcoma. Ann Transl Med 2020;8(6):373. doi: 10.21037/atm.2020.02.74 